\title{
DESENVOLVIMENTO DE MÉTODOS ANALÍTICOS PARA DETERMINAÇÃO DE AGROTÓXICOS EM SEDIMENTOS POR CROMATOGRAFIA GASOSA MONODIMENSIONAL E BIDIMENSIONAL ABRANGENTE COM MICRO DETECTOR DE CAPTURA DE ELÉTRONS
}

\author{
Juliana Macedo da Silva, Cláudia Alcaraz Zini e Elina Bastos Caramão*\# \\ Instituto de Química, Universidade Federal do Rio Grande do Sul, Av. Bento Gonçalves, 9500, 91501-970 Porto Alegre - RS, Brasil \\ Ewelin Monica Paturi Navarro Canizares e Karen Alam Leal \\ Fundação Estadual de Proteção Ambiental Henrique Luiz Roessler, Rua Aurélio Porto, 37, 90620-090 Porto Alegre - RS, Brasil
}

Recebido em 22/3/09; aceito em 19/8/09; publicado na web em 24/2/10

\begin{abstract}
DEVELOPMENT OF ANALYTICAL METHODS FOR PESTICIDES IN SEDIMENTS BY MONODIMENSIONAL AND COMPREHENSIVE TWO-DIMENSIONAL GAS CHROMATOGRAPHY WITH MICRO ELECTRON-CAPTURE DETECTION. The development of analytical methods for determination of eight pesticides of different chemical classes (trichlorfon, propanil, fipronil, propiconazole, trifloxystrobin, permethrin, difenoconazole and azoxystrobin) in sediments with gas chromatographymicro-electron capture detector $(\mathrm{GC} / \mu \mathrm{ECD})$ and comprehensive two-dimensional gas chromatography with micro-electron capture detector $(\mathrm{GCxGC} / \mu \mathrm{ECD})$ is described. These methods were applied to real sediment samples, and the best results were obtained using a 5\% diphenyl-methylpolysiloxane column for 1D-GC. For GCxGC the same column was employed in the first dimension and a 50\%-phenyl-methylpolysiloxane stationary phase was placed in the second dimension. Due to the superior peak capacity and selectivity of GCxGC, interfering matrix peaks were separated from analytes, showing a better performance of GCxGC.
\end{abstract}

Keywords: pesticides; GCxGC; $\mu \mathrm{ECD}$.

\section{INTRODUÇÃO}

O homem vem usufruindo os recursos naturais sem levar em consideração todo o cuidado necessário com sua reposição ou preservação e, com isso, muitos recursos hídricos se encontram em situação precária, seja pela atividade agrícola ou por outras atividades antrópicas. A utilização de agrotóxicos na agricultura vem obtendo cada vez mais destaque no cenário mundial. O Brasil, que até 2007 era o quarto colocado no consumo desses compostos, em 2008 passou a ocupar o segundo lugar, considerando o consumo de dez países que contribuem com $70 \%$ do consumo mundial. ${ }^{1}$

Os agrotóxicos pertencem à classe dos poluentes ambientais mais estudados atualmente e sua determinação em diferentes matrizes se torna cada vez mais necessária, devido à relação direta entre saúde e qualidade ambiental. Nesse contexto, o desenvolvimento de técnicas de separação, identificação e quantificação desses compostos ganha grande destaque para que os limites de detecção e quantificação de tais métodos sejam cada vez menores e contemplem os limites máximos de resíduos (LMR) estabelecidos pelos órgãos competentes. Atualmente, a cromatografia gasosa é a técnica mais utilizada para a determinação desses compostos em diferentes matrizes. ${ }^{2}$

A cromatografia líquida de alta eficiência tem sido intensamente aplicada na pesquisa de resíduos em diferentes matrizes devido à capacidade de detectar compostos termicamente instáveis e não voláteis, que não são identificados por cromatografia gasosa. A literatura científica apresenta alguns artigos de revisão em que o potencial desta técnica acoplada à espectrometria de massas é discutida. Devido ao grande poder de separação para análise de matrizes complexas, a técnica é considerada sensível e seletiva e provê identificação segura em baixas concentrações para diferentes analitos. ${ }^{3,4}$ Porém,

*e-mail: elina@ufrgs.br

\# Instituto Nacional de Ciência e Tecnologia de Energia e Ambiente, UFBAUFRGS para amostras complexas, contendo de 150 a 250 compostos de interesse, por exemplo, a separação obtida por cromatografia gasosa ou líquida, com uma única coluna, não é suficiente para separação de todos os compostos. Outras técnicas multidimensionais como GC-GC (cromatografia gasosa bidimensional de frações parciais ou de heartcut), HPLC-GC (cromatografia líquida de alta eficiência acoplada à cromatografia gasosa) e SFC-GC (cromatografia com fluido supercrítico acoplada à cromatografia gasosa) não produzem também a separação efetiva de todos os analitos. ${ }^{5,6}$

A cromatografia gasosa bidimensional abrangente $(\mathrm{GCxGC})$ é uma técnica relativamente recente, idealizada em 1991 por Phillips ${ }^{7}$ e apresenta grande poder de separação. Na GCxGC, geralmente, duas colunas cromatográficas de diferentes mecanismos de retenção são acopladas em série, sendo a primeira de dimensões convencionais e a segunda mais curta, do tipo fast-GC. O modulador é considerado o "coração" da técnica e é acoplado entre as duas colunas, tendo a função de amostrar e focalizar as estreitas frações eluídas da primeira coluna e, em seguida, liberar estas porções, rapidamente, para a segunda coluna. O termo "abrangente" é utilizado para designar que todo o efluente da primeira dimensão, ou uma parte representativa do mesmo, seja introduzido na segunda dimensão $\left({ }^{2} \mathrm{D}\right)$, sem perda das características da separação na ${ }^{1} \mathrm{D}$ (primeira dimensão). Os princípios da GCxGC, aspectos instrumentais, nomenclatura e aplicações a diversas matrizes encontram-se abordados na literatura científica. ${ }^{8-12}$

Mondello e colaboradores ${ }^{13}$ descrevem a GCxGC como sendo uma das inovações mais revolucionárias na cromatografia gasosa. Os autores também afirmam que, provavelmente, a diferença do poder de separação entre a GCxGC e a 1D-GC (cromatografia gasosa monodimensional) seja ainda maior do que a diferença existente entre as colunas capilares e empacotadas. Em geral, a GCxGC apresenta quatro vantagens sobre a $1 \mathrm{D}-\mathrm{GC}$ : aumento de resolução; aumento de sensibilidade devido à reconcentração da banda do soluto durante o processo de modulação, favorecendo a detecção de componentes em nível de traços; construção de cromatograma ${ }^{2} \mathrm{D}$ (bidimensional) 
com estruturação por compostos quimicamente similares e, aumento da capacidade de pico, pois a retenção nas duas colunas através de diferentes mecanismos de interação analito-fase estacionária, faz com que a capacidade teórica de pico na GCxGC se aproxime do produto das capacidades de pico das duas dimensões. ${ }^{6,13,14}$

Uma consequência importante da utilização dos agrotóxicos na agricultura é a possível presença de resíduos desses compostos e de seus metabólitos em alimentos. Investigação sobre o poder de separação da GCxGC/TOFMS (TOFMS, detector de espectrometria de massas com analisador por tempo de vôo - Time-of-Flight Mass Spectrometry) para determinação de 58 compostos da classe dos organofosforados e nitrogenados em amostras alimentícias foi realizado por Dallüge e colaboradores..$^{15}$ Os autores observaram que a determinação de alguns compostos por 1D-GC/TOFMS seria prejudicada em função da coeluição com interferentes da matriz, já que o espectro de massas obtido pela deconvolução espectral ou pela subtração da linha de base não é claro o suficiente para proporcionar a identificação dos analitos de interesse. Em outro estudo de otimização de metodologia para determinação de 51 agrotóxicos em uva, Banerjee e colaboradores ${ }^{16}$ verificaram que a sensibilidade da análise por GCxGC/TOFMS foi consideravelmente aumentada quando comparada à $1 \mathrm{D}-\mathrm{GC} / \mathrm{TOFMS}$. A GCxGC ainda proporcionou a separação cromatográfica de alguns compostos que coeluem quando analisados por GC/MS; embora estes tenham sido identificados por deconvolução espectral na cromatografia monodimensional. Além disso, a GCxGC possibilita a obtenção de espectros de massas mais nítidos, evitando assim a possível confirmação de falsos positivos. $\mathrm{O}$ aumento de sensibilidade analítica da GCxGC/NPD (NPD, detector de nitrogênio e fósforo) quando comparada à GC/NPD, para análise de vegetais, foi verificado por Khummueng e colaboradores, ${ }^{17}$ visto que a soma das alturas dos picos cromatográficos obtidos pela técnica bidimensional abrangente foi cerca de 20 vezes maior do que as obtidas na técnica monodimensional.

O potencial da GCxGC/ $\mu$ ECD foi verificado por Bordajandi e colaboradores ${ }^{18}$ através de desenvolvimento de método para determinação de poluentes quirais, como toxafeno em óleo de peixe. O método descrito para determinação de cinco congêneros do toxafeno com coluna enantiosseletiva na ${ }^{1} \mathrm{D}$ providenciou completa separação dos isômeros, enquanto que para a 1D-GC, com a mesma coluna enantiosseletiva, ocorreu sobreposição de dois compostos. Além disso, a GCxGC possibilitou excelente separação entre os compostos estudados e os interferentes da matriz complexa.

Korytár e colaboradore ${ }^{19}$ avaliaram a separação de 12 diferentes classes de poluentes, dentre eles agrotóxicos da classe dos organoclorados e toxafeno, por GCxGC/ $\mu \mathrm{ECD}$. Nesse estudo foram utilizados 5 conjuntos de colunas, sendo que em todas as combinações a fase estacionária apolar 100\% dimetilpolissiloxano (DB-1) ou semelhante foi empregada para a separação na ${ }^{1} \mathrm{D}$. A fase estacionária constituída de $65 \%$ fenilmetilpolissiloxano (007-65HT) proporcionou melhor separação para os agrotóxicos organoclorados e também possibilitou a ordenação estrutural dos congêneros do toxafeno, baseada no número de substituintes clorados. A fase estacionária contendo 70 a $90 \%$ de grupos ciano (VF$23 \mathrm{~ms}$ ) proporcionou excelente separação na ${ }^{2} \mathrm{D}$ para os organoclorados e toxafeno, embora não tenha sido possível visualizar a separação de todas as doze classes estudadas. Para avaliar o potencial da técnica, os autores determinaram dibenzodioxinas e dibenzofuranos policlorados (PCDD/F) em amostras de sedimentos e éteres difenílicos polibromados (PBDEs) e alcanos policlorados (PCAs) em amostras de poeira.

O número de publicações científicas contemplando a determinação de agrotóxicos por GCxGC ainda é relativamente pequeno, mas suficiente para demonstrar as potenciais vantagens da técnica em relação à 1D-GC. ${ }^{20}$ Não é do conhecimento destes autores a existência de artigos publicados envolvendo a determinação de agrotóxicos por $\mathrm{GCxGC/}$ $\mu \mathrm{ECD}$ em sedimentos.
Este estudo tem por objetivo o desenvolvimento de métodos analíticos por $1 \mathrm{D}-\mathrm{GC} / \mu \mathrm{ECD}$ e GCxGC/ $\mu \mathrm{ECD}$ para a determinação de oito agrotóxicos de diferentes classes químicas em sedimentos de rios provenientes de regiões sob influência da cultura do arroz, utilizados no estado do Rio Grande do Sul, uma vez que o mesmo é responsável por $61 \%$ da produção deste cereal no país. ${ }^{1}$ Estes níveis de produção são alcançados através do intenso uso de agrotóxicos durante o plantio.

\section{PARTE EXPERIMENTAL}

\section{Os sedimentos e agrotóxicos estudados}

Para os estudos qualitativos, como verificação da complexidade da matriz, foram analisadas duas amostras de sedimentos de pontos localizados em diferentes bacias hídricas: Rio Gravataí (latitude sul $=29^{\circ} 56^{\prime} 10^{\prime \prime}$ e longitude oeste $=50^{\circ} 36^{\prime}$ 05") e Rio Santa Maria (latitude sul $=30^{\circ} 52^{\prime} 15^{\prime \prime}$ e longitude oeste $=55^{\circ} 20^{\prime}$ '57'). Foram coletados em média $1 \mathrm{~kg}$ de sedimento com auxílio de pá de aço inoxidável, próximo à margem e em locais de deposição de partículas finas e de pouca profundidade. Os sedimentos foram armazenados em frascos de vidro e transportados a $4{ }^{\circ} \mathrm{C}$ até o laboratório, onde foram congelados $\mathrm{a}-18^{\circ} \mathrm{C}$ em freezer antes do processo de extração.

Os compostos estudados foram triclorfom, propanil, fipronil, propiconazol, trifloxistrobina, permetrina, difenoconazol e azoxistrobina (pureza $>97 \%$, Sigma-Aldrich, Seelze, Alemanha). Estes agrotóxicos são largamente empregados na agricultura, mais especificamente na cultura do arroz da região do Rio Santa Maria no estado do Rio Grande do Sul. ${ }^{21,22}$ A química Ewelin Canizares, da FEPAM, realizou pesquisa com os agricultores e com os trabalhadores que fazem a aplicação desses compostos na lavoura, bem como com a EMBRAPA para embasar a escolha dos agrotóxicos. A Tabela 1 apresenta algumas características e estruturas químicas dos compostos estudados.

\section{Extração das amostras de sedimentos}

O processo de extração empregado está baseado no método reportado por You e colaboradores. ${ }^{23} \mathrm{~A}$ água residual presente no sedimento foi retirada e o mesmo foi homogeneizado. Em seguida, foi parcialmente seco ao ar, à temperatura ambiente, em vidros de relógio. Para a etapa de extração, foram pesados $20 \mathrm{~g}$ de sedimento e adicionados a este, $40 \mathrm{~g}$ de sulfato de sódio anidro (Quimex, SP, Brasil), procedendo-se à homogeneização do material. Uma alíquota de $50 \mathrm{~mL}$ de uma solução 50:50 v/v de acetona:diclorometano foi adicionada à amostra, e esta foi submetida à extração em 3 ciclos de 15 min no banho de ultrassom Maxiclean (Unique, Indaiatuba, SP, Brasil) com potência de 120 W. A etapa de purificação (clean up) foi realizada em colunas de vidro de $30 \mathrm{~cm}$ de altura por $14 \mathrm{~mm}$ de diâmetro interno, contendo $10 \mathrm{~g}$ de Florisil (60-100 mesh; T.J. Baker, Phillipsburg, EUA) ativado a $90{ }^{\circ} \mathrm{C}$ por $12 \mathrm{~h} \mathrm{e}$, posteriormente, parcialmente desativado com água destilada $(6 \% \mathrm{~m} / \mathrm{v})$. No topo da coluna foi colocado aproximadamente $1 \mathrm{~cm}$ de sulfato de sódio anidro para reter qualquer umidade residual. A eluição dos analitos foi realizada com $50 \mathrm{~mL}$ de solução $30 \%$ de éter etílico em hexano. O extrato foi levado à secura e o resíduo final dissolvido com 1,5 mL de hexano. Todos os solventes utilizados para extração eram de grau analítico (Vetec, Rio de Janeiro, RJ, Brasil) e bidestilados.

\section{Desenvolvimento dos métodos cromatográficos}

As análises por 1D-GC e GCxGC foram realizadas em um cromatógrafo Agilent 6890N (Agilent Technologies, Palo Alto, CA, EUA) com $\mu E C D$, equipado com amostrador automático Combi PAL (CTC 
Tabela 1. Agrotóxicos estudados, uso, grupo químico e fórmula estrutural

\begin{tabular}{lcc}
\hline Nome & Uso & Grupo Químico \\
\hline Triclorfom (1) & Inseticida & Organofosforado \\
Propanil (2) & Herbicida & Anilida \\
Fipronil (3) & Inseticida & Pirazol \\
Propiconazol (4) & Fungicida & Triazol \\
Trifloxistrobina (5) & Fungicida & Estrobilurina \\
Permetrina (6) & Inseticida & Piretróide \\
Difenoconazol (7) & Fungicida & Triazol \\
Azoxistrobina $(\mathbf{8})$ & Fungicida & Estrobilurina \\
\hline
\end{tabular}

$$
{ }_{1}
$$<smiles>CCCC1COC(Cn2cncn2)(c2ccc(Cl)cc2Cl)O1</smiles><smiles>CO/N=C(/C(=O)OC)c1ccccc1CO/N=C(\C)c1cccc(C(F)(F)F)c1</smiles><smiles>CC(C)(C=C(Cl)Cl)C(=O)OCc1cccc(Oc2ccccc2)c1</smiles><smiles>CC1COC(Cn2cncn2)(c2ccc(Oc3ccc(Cl)cc3)cc2Cl)O1</smiles><smiles>CO/C=C(/C(=O)OC)c1ccccc1Oc1cc(Oc2ccccc2C#N)ncn1</smiles>

Analytics AG, Zwingen, Suíça). O cromatógrafo também possui um forno secundário (Leco, St. Joseph, MI, EUA) utilizado nas análises por GCxGC e um modulador térmico de dois estágios com quatro jatos (quad jet) de $\mathrm{N}_{2}$ (dois jatos quentes e dois frios que são resfriados por $\mathrm{N}_{2}$ líquido). Durante o desenvolvimento dos métodos foram utilizadas colunas cromatográficas de diferentes fases estacionárias, que estão apresentadas na Tabela 2. Outros parâmetros experimentais que influenciam na análise dos compostos estudados, tais como programação de temperatura do forno, temperatura do injetor $\left(250,280\right.$ e $\left.300{ }^{\circ} \mathrm{C}\right) \mathrm{e}$ fluxo de gás de make up $\left(\mathrm{N}_{2}, 99,999 \%\right.$, Linde Gás) foram otimizados para a análise por GC/ $\mu$ ECD. O fluxo de gás de arraste $\left(\mathrm{H}_{2}, 99,999 \%\right.$, Linde Gás) utilizado nos experimentos foi de $2 \mathrm{~mL} \mathrm{~min}^{-1}$. A partir dos melhores resultados obtidos para a $1 \mathrm{D}-\mathrm{GC}$, parâmetros como período de modulação, diferença de temperatura entre forno primário e secun- dário e duração do jato quente foram otimizados para as análises por GCxGC/ $\mu$ ECD, utilizando-se diferentes jogos de colunas.

Para a verificação dos tempos de retenção dos analitos, foi realizada a injeção de solução padrão contendo os nove compostos (oito agrotóxicos e um padrão interno - 3,4,5-tricloroguaiacol para posterior estudo quantitativo), na concentração de $100 \mu \mathrm{g} \mathrm{L}^{-1}$. As soluções padrão estoque foram preparadas pesando-se os agrotóxicos em frascos individuais em balança analítica de precisão (Shimadzu, AY220, Quioto, Japão) e dissolvendo-os em acetato de etila grau HPLC (Mallincrrodt, Phillipsburg, EUA) em balões volumétricos de $5 \mathrm{~mL}$, previamente silanizados. As diluições posteriores foram também feitas em balões volumétricos de $5 \mathrm{~mL}$ e em acetato de etila, utilizando-se micropipeta (Brand, Wertheim, Alemanha) para coleta das alíquotas. Todas as soluções foram guardadas em freezer à $-18^{\circ} \mathrm{C}$ e em frascos de vidro âmbar silanizados. As injeções foram realizadas com volume de $1 \mu \mathrm{L}$, sem divisão de fluxo e com pulso de pressão de 60 psi. Durante todas as análises, a temperatura do detector foi mantida entre $20-25^{\circ} \mathrm{C}$ acima da temperatura mais alta do forno, a fim de evitar e/ou diminuir o efeito de cauda nos picos e manter a célula do detector limpa. ${ }^{24}$

\section{RESULTADOS E DISCUSSÃO}

\section{Cromatografia gasosa monodimensional (1D-GC)}

Inicialmente foram feitos testes para verificar a influência do gás de make up $\left(\mathrm{N}_{2}\right)$ na resposta do detector aos diferentes agrotóxicos, empregando-se para isto a coluna HP-50+. Os fluxos testados foram de 30 e $60 \mathrm{~mL} \mathrm{~min}^{-1}$, que são os recomendados pelo manual do fabricante. ${ }^{24}$ A condição de $30 \mathrm{~mL} \mathrm{~min}^{-1}$ foi considerada a mais apropriada devido à obtenção das melhores intensidades de sinal para os agrotóxicos em estudo e, por esta razão, nos experimentos posteriores essa condição foi mantida. A diminuição de fluxo de gás de make up faz com que o efeito de diluição dos compostos no detector seja minimizado, com consequente diminuição na largura da base dos picos, proporcionando melhor desempenho no processo cromatográfico. A partir dessa condição foram realizadas injeções sem divisão de fluxo com pulso de pressão de 60 psi com diferentes períodos de duração, a fim de se obter uma transferência mais rápida da banda cromatográfica para o interior da coluna, bem como o consequente estreitamento e aumento de intensidade da mesma. ${ }^{25} \mathrm{~A}$ literatura reporta algumas vantagens da utilização da injeção com pulso de pressão, tais como o aumento da velocidade de transferência de analitos com elevado ponto de ebulição e de compostos que possam ser adsorvidos no injetor, redução do tempo de residência da amostra no injetor, aumento da capacidade do mesmo, em função do volume de vapor ser inversamente proporcional à pressão. ${ }^{26}$ Verificou-se que a eluição do triclorfom durante o pulso de pressão faz com que a intensidade de sinal deste pico aumente desproporcionalmente em relação aos demais analitos, podendo também ocorrer coeluição do triclorfom com o pico cromatográfico do solvente. A escolha de um pulso de pressão cuja duração seja apropriada (36,6 s para HP-50+ e DB-WAXetr; 29,4 s para DB-5) resulta em um gerenciamento adequado destes fenômenos, proporcionando uma condição cromatográfica satisfatória. As três colunas utilizadas para os experimentos apresentam dimensões idênticas, mas fases estacionárias diferenciadas, o que implica em tempos de retenção distintos para os analitos e solvente em cada fase.

Outras duas fases estacionárias foram utilizadas na 1D-GC e diversos testes de programação de temperatura do forno foram testados. A Tabela 3 apresenta as condições analíticas que resultaram na melhor performance cromatográfica para cada coluna capilar empregada. A DB-5 foi a coluna cromatográfica que permitiu a melhor separação de todos os compostos em um menor tempo de análise. 
Tabela 2. Colunas utilizadas nas otimizações dos parâmetros cromatográficos paxa 1D-GC e GCxGC

\begin{tabular}{lccccc}
\hline $\begin{array}{l}\text { Conj. de } \\
\text { Colunas }\end{array}$ & \multicolumn{2}{c}{ Primeira Dimensão ( $\left.{ }^{1} \mathrm{D}\right)$ e para 1D-GC* } & \multicolumn{2}{c}{ Segunda Dimensão $\left({ }^{2} \mathrm{D}\right)$} \\
\hline$(1)$ & Nome & Fase estacionária & Nome & Fase estacionária & Dimensões \\
$(2)$ & DB-5 & $5 \%$ difenil-95\% dimetilpolissiloxano & DB- $17 \mathrm{~ms}$ & $50 \%$ fenil-50\% metilpolissiloxano & $1,70 \mathrm{~m} \times 0,18 \mathrm{~mm} \times 0,18 \mu \mathrm{m}$ \\
\hline
\end{tabular}

* As dimensões das colunas empregadas em $1 \mathrm{D}-\mathrm{GC}$ ou na ${ }^{1} \mathrm{D}$ foram sempre $30 \mathrm{~m}$ x $0,25 \mathrm{~mm}$ x $0,25 \mu \mathrm{m}$. A coluna DB-WAXetr possui fase estacionária constituída por $100 \%$ polietilenoglicol.

Tabela 3. Condições cromatográficas obtidas para 1D-GC

\begin{tabular}{|c|c|c|c|}
\hline \multirow{2}{*}{ Condições } & \multicolumn{3}{|c|}{ Colunas cromatográficas } \\
\hline & HP-50+ & DB-5 & DB-WAXetr \\
\hline $\begin{array}{l}\text { Programação de } \\
\text { Temperatura (T) do Forno }\end{array}$ & $\begin{array}{c}50{ }^{\circ} \mathrm{C}(1,5 \mathrm{~min})-30^{\circ} \mathrm{C} \min ^{-1} / 190{ }^{\circ} \mathrm{C}-5^{\circ} \mathrm{C} \\
\min ^{-1} / 220^{\circ} \mathrm{C}-7^{\circ} \mathrm{C} \min ^{-1} / 255^{\circ} \mathrm{C}(44 \mathrm{~min})\end{array}$ & $\begin{array}{c}60^{\circ} \mathrm{C}(2,53 \mathrm{~min})-35^{\circ} \mathrm{C} \mathrm{min}^{-1} / 180^{\circ} \mathrm{C} \\
-4{ }^{\circ} \mathrm{C} \min ^{-1} / 240{ }^{\circ} \mathrm{C}-12^{\circ} \mathrm{C} \mathrm{min}^{-1} / 300{ }^{\circ} \mathrm{C}(2 \\
\min )\end{array}$ & $\begin{array}{c}40^{\circ} \mathrm{C}(1,5)-12^{\circ} \mathrm{C} \mathrm{min}^{-1} / 100{ }^{\circ} \mathrm{C}-30 \\
{ }^{\circ} \mathrm{C} \min ^{-1} / 235^{\circ} \mathrm{C}(44 \mathrm{~min})\end{array}$ \\
\hline $\mathrm{T}$ do Injetor $\left({ }^{\circ} \mathrm{C}\right)$ & 280 & 280 & 260 \\
\hline $\mathrm{T}$ do Detector $\left({ }^{\circ} \mathrm{C}\right)$ & 280 & 325 & 260 \\
\hline Pulso de Pressão (s) & 36,6 & 29,4 & 36,6 \\
\hline
\end{tabular}

Sabendo-se que a DB-5 apresenta maior resistência a temperaturas elevadas do que a HP-50+ $\left(325\right.$ e $280{ }^{\circ} \mathrm{C}$ em condição isotérmica, respectivamente), foram realizados testes com três diferentes temperaturas de injeção - 250, 280 e $300{ }^{\circ} \mathrm{C}$ - a fim de verificar a influência deste parâmetro no sinal cromatográfico obtido para os compostos em estudo. De acordo com a Figura 1, os dados indicaram que, de forma geral, não há diferença significativa nas alturas relativas dos picos nas temperaturas de 280 e $300{ }^{\circ} \mathrm{C}$. Contudo, a temperatura de $280{ }^{\circ} \mathrm{C}$ foi escolhida por ter propiciado maior intensidade do sinal cromatográfico do componente fipronil. Zhang e colaboradores ${ }^{27}$ também verificaram que ocorre uma melhora do sinal cromatográfico para vários analitos, quando da utilização de temperaturas mais elevadas no injetor.

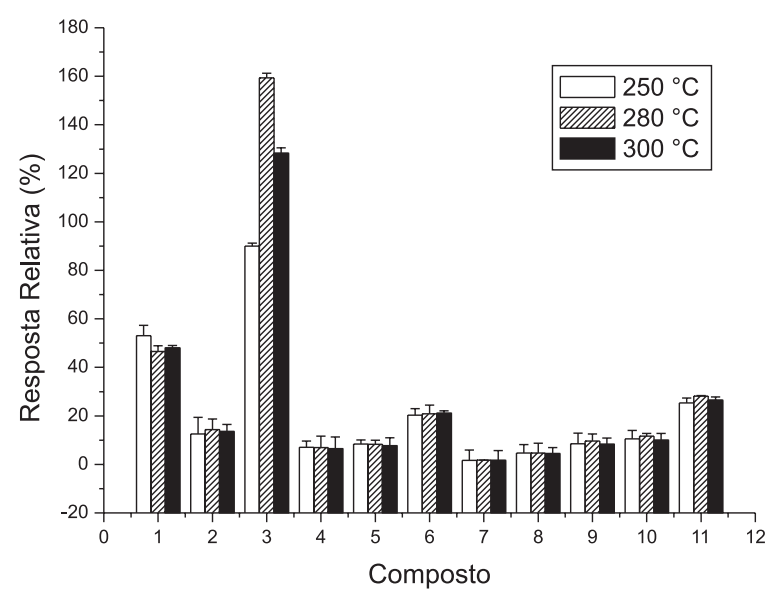

Figura 1. Comparação das médias das respostas relativas dos picos cromatográficos (n=3) para diferentes temperaturas de injeção. 1) Triclorfom, 2) propanil, 3) fipronil, 4) propiconazol I, 5) propiconazol II, 6) trifloxistrobina, 7 e 8) permetrina cis e trans, 9) difenoconazol I, 10) difenoconazol II e 11) azoxistrobina

Em função da baixa temperatura máxima de trabalho em condição isotérmica para a coluna DB-WAXetr, de fase estacionária $100 \%$ polietilenoglicol, observou-se que, mesmo após $180 \mathrm{~min}$ a $235{ }^{\circ} \mathrm{C}$, não houve a eluição de dois dos compostos - difenoconazol e azoxistrobina - os quais possuem massas moleculares maiores do que as dos demais analitos (406,2 e 403,4 $\mathrm{g} \mathrm{mol}^{-1}$, respectivamente). Tal fato inviabiliza o uso da DB-WAXetr. Além da dificuldade de eluição destes compostos, constatou-se que essa coluna possivelmente provocou sorção parcial dos compostos, devido à sua alta polaridade. Isto pode ser observado através da baixa intensidade dos sinais cromatográficos, quando comparados àqueles obtidos em análises feitas com as mesmas soluções em outras fases estacionárias.

O uso da DB-5 oferece vantagens em relação à HP-50+, pois além de proporcionar menor tempo de análise na separação dos nove compostos (27,9 e 61,1 min, respectivamente), ainda oferece melhor resolução na separação dos picos cromatográficos, com exceção do propiconazol II e do trifloxistrobina que apresentaram resolução de 1,26 e 2,04 para DB-5 e HP-50+, respectivamente. A coluna DB-5 também proporcionou a separação dos dois isômeros do difenoconazol com resolução de 0,89 , enquanto que na HP-50+ estes dois isômeros coeluem. Isto se deve às diferenças nas interações entre analito e fase estacionária e também ao fato de a DB-5 permitir o emprego de uma temperatura máxima maior $\left(300{ }^{\circ} \mathrm{C}\right)$ na análise cromatográfica do que a temperatura máxima da $\mathrm{HP}-50+\left(255^{\circ} \mathrm{C}\right)$.

$\mathrm{O}$ fator de assimetria $\left(\mathrm{A}_{\mathrm{s}}\right)$ de um pico cromatográfico está diretamente relacionado à eficiência cromatográfica. Fatores de assimetria entre 0,8 e 1,2 são considerados satisfatórios, com pouca ou nenhuma influência de cauda nos picos cromatográficos. Tanto a HP-50+ quanto a DB-5 apresentaram, em geral, valores satisfatórios de assimetria. A Tabela 4 apresenta os fatores de assimetria para os picos cromatográficos e os tempos de retenção obtidos para os compostos estudados. Esse fator foi calculado utilizando-se a razão entre as larguras B e A a 10\% da altura do pico, conforme Figura 2. ${ }^{28}$

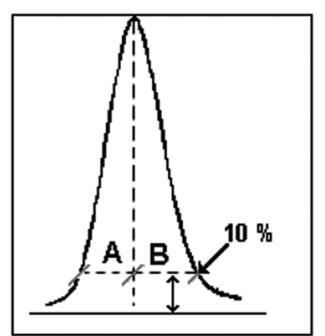

Figura 2. Representação de um pico cromatográfico genérico, ilustrando os parâmetros empregados para cálculo do fator de assimetria

À luz dos resultados obtidos para os vários testes realizados com as três colunas cromatográficas, a DB-5 foi escolhida por sua melhor performance. 
Tabela 4. Tempos de retenção $\left(t_{R}\right)$, fatores de assimetria $\left(A_{s}\right)$ e desvio padrão (DP) obtidos para os compostos estudados com as diferentes colunas cromatográficas

\begin{tabular}{lcccc}
\hline \multirow{2}{*}{ Analito } & \multicolumn{2}{c}{$\mathrm{HP}-50+$} & \multicolumn{2}{c}{$\mathrm{DB}-5$} \\
& $\mathrm{t}_{\mathrm{R}}(\mathrm{min})$ & $\mathrm{A}_{\mathrm{s}}(\mathrm{DP})$ & $\mathrm{t}_{\mathrm{R}}(\mathrm{min})$ & $\mathrm{A}_{\mathrm{s}}(\mathrm{DP})$ \\
\hline Triclorfom & 0,77 & $1,4(0,07)$ & 0,59 & $2,0(0,87)$ \\
Tricloroguaiacol (PI) & 7,74 & $1,5(0,29)$ & 7,46 & $1,0(0,24)$ \\
Propanil & 10,93 & $1,0(0,00)$ & 9,90 & $1,5(0,29)$ \\
Fipronil & 11,77 & $0,8(0,14)$ & 12,59 & $1,0(0,19)$ \\
Propiconazol I & 16,54 & $1,0(0,19)$ & 16,75 & $1,0(0,00)$ \\
Propiconazol II & 16,63 & $1,3(0,34)$ & 16,98 & $1,3(0,33)$ \\
Trifloxistrobina & 16,87 & $1,0(0,14)$ & 17,14 & $1,1(0,23)$ \\
Permetrina I & 21,79 & $0,9(0,08)$ & 22,43 & $1,0(0,14)$ \\
Permetrina II & 22,19 & $1,0(0,07)$ & 22,65 & $1,0(0,19)$ \\
*Difenoconazol I & 36,80 & - & 25,24 & $1,5(0,00)$ \\
*Difenoconazol II & - & - & 25,31 & $1,0(0,24)$ \\
Azoxistrobina & 57,35 & $1,1(0,07)$ & 25,89 & $0,7(0,19)$ \\
\hline
\end{tabular}

*O fator de assimetria para o difenoconazol, com a utilização da coluna HP-50+, não foi calculado em função da coeluição parcial entre os isômeros.

\section{Cromatografia gasosa bidimensional abrangente $(\mathrm{GC} \times \mathrm{GC})$}

De acordo com Kristenson e colaboradores, ${ }^{29}$ quanto maior o fluxo

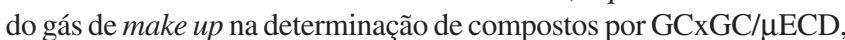
menor é o efeito de cauda observado nos picos cromatográficos. Contudo, o efeito de diluição dos compostos no detector é maior. Tendo por base os resultados destes pesquisadores e também de outros, ${ }^{18,30} \mathrm{O}$ fluxo de nitrogênioutilizado para todos os experimentos foi de $150 \mathrm{~mL}$ $\min ^{-1}$, que é o fluxo máximo permitido pelo equipamento. A Tabela 5 apresenta as melhores condições de análise obtidas para a GCxGC.

Parâmetros como período de modulação e duração dos jatos quentes foram testados para todos os conjuntos de colunas. Os máximos períodos testados para a duração do jato quente foram de $30 \%$ do período de modulação. A Tabela 6 apresenta os tempos de retenção na primeira e segunda dimensões, obtidos para os analitos com a utilização dos diferentes jogos de colunas, bem como os fatores de assimetria encontrados para as diferentes durações de jato quente. $\mathrm{O}$ texto a seguir discute os resultados obtidos.

\section{Conjunto de colunas n⿳1 1 (DB-5/DB-17ms)}

Inicialmente, foram testados diferentes períodos de modulação $\left(P_{M}\right): 2,4,6,7$ e 8 s. Destes, foi escolhido $7 \mathrm{~s}$, pois neste $P_{M}$ foi observada a melhor distribuição de picos no diagrama de cores. A duração do jato quente é um fator importante na análise, pois pode influenciar no formato e intensidade dos picos. ${ }^{31}$ Com isso, foram testadas diferentes durações do jato, tais como 0,6 s (valor padrão de operação do equipamento); 1,$4 ; 1,75$ e 2,1 s. Os fatores de assimetria para esse conjunto de colunas foram calculados levando-se em consideração o pico modulado mais intenso. ${ }^{17}$ Para uma duração de $0,6 \mathrm{~s}$, a simetria dos picos no diagrama de cores é bastante distorcida, fazendo com que ocorra coeluição parcial entre alguns compostos e, portanto, o fator de assimetria não foi calculado para esse parâmetro. Com a utilização de uma duração de jato quente de $2,1 \mathrm{~s}$, houve melhoria da simetria dos picos relativamente aos demais períodos testados. Cabe acrescentar que as diferentes durações de jato quente testadas não contribuíram com aumento de sinal analítico dos compostos.

A diferença de temperatura utilizada entre o forno primário e secundário foi mantida em $5{ }^{\circ} \mathrm{C}$, visto que, devido aos máximos de temperatura permitidos para cada coluna, não foi possível variar este parâmetro.

\section{Conjunto de colunas n ${ }^{\circ} 2$ (HP-50+/DB-1ms)}

Os períodos de modulação testados foram de 2,4 e $6 \mathrm{~s}$ e as diferenças de temperaturas entre os fornos foram de 5, 20, 30 e $40{ }^{\circ} \mathrm{C}$. Devido à maior resistência de temperatura da coluna da ${ }^{2} \mathrm{D}$ (DB-1ms), o método anteriormente utilizado para 1D-GC foi modificado ao final para uma isoterma a $280^{\circ} \mathrm{C}$ (temperatura limite da HP-50+, no modo isotérmico) com duração de $30 \mathrm{~min}$. Esse aumento de temperatura possibilitou diminuição no tempo total de análise em relação à 1D-GC, já que os compostos eluíram em menor tempo de retenção. $\mathrm{O} P_{M}$ que resultou em melhor distribuição dos picos cromatográficos no espaço de separação foi o de $4 \mathrm{~s}$. A variação da diferença de temperatura entre os fornos não contribuiu para melhor separação dos compostos e também não favoreceu o aumento da intensidade de sinal dos picos cromatográficos. Entretanto, constatou-se que com uma diferença de $5{ }^{\circ} \mathrm{C}$ entre o forno primário e o secundário, os analitos distribuíram-se amplamente no espaço de separação, de forma a melhor aproveitá-lo.

Um terceiro parâmetro otimizado foi a duração do jato quente. Os valores testados foram de 0,$6 ; 0,8 ; 1 ;$ e $1,2 \mathrm{~s}$. De forma geral, não houve melhoria na simetria dos picos, quando se alterou a duração do jato quente, sendo que esse fator foi calculado da mesma forma como para o conjunto de colunas $\mathrm{n}^{\circ} 1$. Nenhum dos valores testados contribuiu para a obtenção de bons resultados para a simetria. Em relação à altura dos picos, as maiores intensidades foram obtidas com a utilização dos jatos quentes com durações de 0,8 e 1,2 s, sendo que as alturas observadas foram muito próximas para esses dois valores. A duração de $0,8 \mathrm{~s}$ foi escolhida em função dos valores de assimetria obtidos serem mais próximos dos valores aceitáveis e por apresentar menor desvio padrão, em comparação com os outros valores testados.

\section{Comparação entre os jogos de colunas DB-5/DB-17ms e HP- 50+/DB-1ms}

O conjunto de colunas $\mathrm{n}^{\circ} 1$ (DB-5/DB-17ms) proporcionou melhores resultados analíticos em relação ao $\mathrm{n}^{\circ} 2$ (HP-50+/DB-1ms) devido ao menor tempo de análise $\left(29,4\right.$ min para o conjunto $\mathrm{n}^{\circ} 1 \mathrm{e}$ 35,7 min para o conjunto $n^{\circ} 2$ ) e melhor resolução para permetrina $\left(\mathrm{R}_{\mathrm{s}}=0,72\right.$ para o conj. $\mathrm{n}^{\circ} 1$ e $\mathrm{R}_{\mathrm{s}}=0,36$ para o conj. $\left.\mathrm{n}^{\circ} 2\right)$. A resolução entre o propiconazol e trifloxistrobina foi de 0,6 para os dois jogos de colunas. Diferentemente da 1D-GC, na GCxGC mais de dois picos

Tabela 5. Condições cromatográficas obtidas para GCxGC

\begin{tabular}{lcccc}
\hline $\begin{array}{l}\text { Conj. } \\
\text { Colunas }\end{array}$ & Programação do Forno & Temperatura $\left({ }^{\circ} \mathrm{C}\right)$ & \multicolumn{2}{c}{ Tempo (s) } \\
\hline$(1)$ & Injetor & Detector & $\Delta \mathrm{T}$ entre os Fornos & $\mathrm{P}_{\mathrm{M}}$ \\
Jato Quente
\end{tabular}

A temperatura do modulador foi mantida a $20^{\circ} \mathrm{C}$ acima da temperatura do forno primário. $\mathrm{P}_{\mathrm{M}}$ é o período de modulação em segundos. 
Tabela 6. Tempos de retenção, fatores de assimetria $\left({ }^{2} A_{s}\right)$ e desvio padrão para os picos na ${ }^{2} \mathrm{D}$ com a utilização de diferentes durações de jato quente

$$
\text { DB-5/DB-17ms }
$$

HP-50+/DB-1ms

\begin{tabular}{|c|c|c|c|c|c|c|c|c|c|c|}
\hline \multirow{3}{*}{$\begin{array}{l}\text { Analito } \\
\text { Triclorfom }\end{array}$} & \multirow{2}{*}{\multicolumn{2}{|c|}{${ }^{1} t_{R}(\min )^{2} t_{R}(\mathrm{~s})$}} & \multicolumn{6}{|c|}{ Duração dos jatos quentes (s) / Desvio padrão } & \multirow{2}{*}{\multicolumn{2}{|c|}{${ }^{1} t_{R}(\min )^{2} t_{R}(\mathrm{~s})$}} \\
\hline & & & \multirow{2}{*}{$\frac{1,4}{1,2 / 0,08}$} & \multirow{2}{*}{$\frac{1,75}{1,1 / 0,00}$} & \multirow{2}{*}{$\frac{2,1}{1,8 / 0,14}$} & \multirow{2}{*}{$\frac{0,8}{1,9 / 0,46}$} & \multirow{2}{*}{$\frac{1,0}{1,7 / 0,46}$} & \multirow{2}{*}{$\frac{1,2}{1,4 / 0,00}$} & & \\
\hline & 2,80 & 2,06 & & & & & & & 2,20 & 1,58 \\
\hline Tricloroguaiacol (PI) & 7,93 & 3,16 & $1,8 / 0,11$ & $2,1 / 0,38$ & $1,4 / 0,21$ & $2,7 / 0,87$ & $3,4 / 0,87$ & $2,9 / 0,62$ & 7,60 & 1,80 \\
\hline Propanil & 10,61 & 5,94 & $1,5 / 0,03$ & $1,6 / 0,03$ & $1,1 / 0,31$ & $3,6 / 0,29$ & $3,9 / 0,29$ & $3,6 / 0,48$ & 10,80 & 2,12 \\
\hline Fipronil & 13,41 & 4,94 & $1,3 / 0,07$ & $1,3 / 0,07$ & $1,3 / 0,03$ & $1,5 / 0,00$ & $1,5 / 0,00$ & $2,0 / 0,28$ & 11,66 & 2,76 \\
\hline Propiconazol I & 17,85 & 1,36 & $1,3 / 0,11$ & $1,4 / 0,07$ & $1,2 / 0,12$ & $1,9 / 0,36$ & $1,9 / 0,36$ & $2,0 / 0,12$ & 16,60 & 2,40 \\
\hline Propiconazol II & 18,08 & 1,30 & $1,3 / 0,10$ & $1,4 / 0,03$ & $1,2 / 0,06$ & - & - & - & - & - \\
\hline Trifloxistrobina & 18,2 & 0,90 & $1,1 / 0,07$ & $1,1 / 0,11$ & $1,2 / 0,10$ & $2,1 / 0,36$ & $2,0 / 0,36$ & $1,8 / 0,21$ & 16,86 & 2,16 \\
\hline Permetrina I & 23,33 & 6,00 & $1,3 / 0,03$ & $1,3 / 0,10$ & $1,2 / 0,09$ & $1,8 / 0,18$ & $1,9 / 0,18$ & $1,9 / 0,16$ & 20,53 & 2,26 \\
\hline Permetrina II & 23,56 & 5,84 & $1,1 / 0,03$ & $1,2 / 0,03$ & $1,2 / 0,08$ & $2,4 / 0,00$ & $2,4 / 0,00$ & $1,9 / 0,13$ & 20,73 & 2,24 \\
\hline Difenoconazol & 26,36 & 5,94 & $1,3 / 0,03$ & $1,5 / 0,08$ & $1,3 / 0,04$ & $2,1 / 0,17$ & $1,9 / 0,17$ & $2,2 / 0,26$ & 26,80 & 3,22 \\
\hline Azoxistrobina & 27,18 & 1,40 & $1,3 / 0,12$ & $1,3 / 0,06$ & $1,2 / 0,11$ & $2,2 / 0,23$ & $2,3 / 0,36$ & $2,2 / 0,20$ & 34,06 & 3,44 \\
\hline
\end{tabular}

podem ser considerados como vizinhos de um determinado analito e este novo conceito de vizinhança foi introduzido por Schure, ${ }^{32}$ que foi o primeiro autor a propor a abordagem relacionada à teoria da razão vale-pico para cromatografia bidimensional. Para tanto, utiliza-se a distância entre os máximos de dois picos, o ponto de menor intensidade na reta entre estes dois picos e os valores de intensidade nesses três pontos. Contudo, essa abordagem é válida apenas para picos gaussianos. Peters e colaboradores ${ }^{33}$ propuseram uma abordagem para cálculo de resolução baseada na teoria da razão vale-pico (monodimensional), a qual pode ser aplicada tanto para picos gaussianos, quanto não gaussianos. Por outro lado, Adam e colaboradores $^{34,35}$ calcularam a resolução em GCxGC como sendo a média euclidiana das resoluções em cada uma das duas dimensões. Neste trabalho, as resoluções foram calculadas de acordo com esta última abordagem.

Em relação ao fator de assimetria dos picos, o conjunto $n^{\circ} 1$ apresentou melhores resultados, com valores entre 1,1 e 1,8 para o jato quente de $2,1 \mathrm{~s}$, sendo que apenas quatro compostos apresentaram valores de assimetria acima de 1,2 (triclorfom, tricloroguaiacol, fipronil e difenoconazol). $\mathrm{O}$ uso do conjunto $\mathrm{n}^{\circ} 2$ não resultou em fatores de assimetria satisfatórios, apresentando valores entre 1,5 a 3,6 . Apesar do conjunto $n^{\circ} 1$ ter proporcionado maior aproveitamento do espaço de separação, o conjunto $\mathrm{n}^{\circ} 2$ apresentou picos mais estreitos na ${ }^{2} \mathrm{D}$, o que resulta em aumento de sensibilidade pelo aumento na intensidade de sinal analítico e, eventualmente, aumento na capacidade de pico. Os picos modulados foram cerca de 8 a $62 \%$ mais estreitos que os obtidos para o conjunto ${ }^{\circ} 1$, com exceção do triclorfom e propanil, que apresentaram picos mais largos, de 31 e $15 \%$, respectivamente.

A maioria dos trabalhos reportados na literatura apresenta o jogo convencional de colunas (fase estacionária apolar na ${ }^{1} \mathrm{D}$ e polar na $\left.{ }^{2} \mathrm{D}\right)$ como sendo o que proporciona melhores resultados de análise para agrotóxicos em diversas matrizes. ${ }^{16,36}$ Khummueng e colaboradore ${ }^{17}$ avaliaram três conjuntos de colunas para determinação de fungicidas em vegetais, sendo dois conjuntos de geometria inversa (fase estacionária polar ou de média polaridade na ${ }^{1} \mathrm{D}$ e apolar na $\left.{ }^{2} \mathrm{D}\right)$ e um conjunto de geometria convencional. O conjunto de colunas convencional foi considerado o mais eficiente, pois resultou na separação de todos os compostos estudados e em bons formatos de pico. Entretanto, Bordajandi e colaboradore ${ }^{30}$ verificaram que, para a determinação de organoclorados, os melhores resultados de separação foram obtidos com conjunto de colunas polar versus não polar, apesar da restrição de ortogonalidade caracterizada pela utilização de um conjunto inverso de colunas. Em concordância com a maior parte dos estudos relatados anteriormente, o conjunto de colunas $\mathrm{n}^{\circ} 1$ (DB-5/DB-17ms) apresentou melhor aproveitamento do espaço de separação, com ampla distribuição dos analitos na ${ }^{2} \mathrm{D}$, o que é uma característica importante e desejável quando da análise de amostras complexas.

\section{Análise de amostras reais de sedimentos}

A aplicabilidade dos métodos por $1 \mathrm{D}-\mathrm{GC}$ com a coluna DB-5 e GCxGC com o jogo de colunas n ${ }^{\circ} 1$ (DB-5/DB-17ms) foi investigada através da análise de duas amostras de sedimentos. Em nenhuma das amostras foi verificada a presença dos analitos estudados, mas percebe-se claramente, através do cromatograma de uma amostra fortificada com compostos padrão (Figura 3), que a análise por 1D-GC traria prejuízo à quantificação, caso fossem encontrados os analitos nas amostras, devido à coeluição dos mesmos com constituintes da matriz.

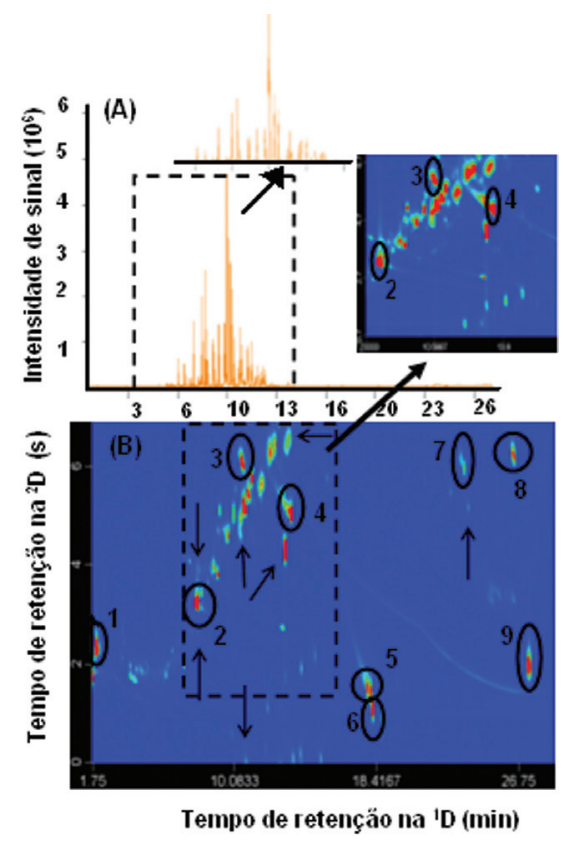

Figura 3. Cromatogramas do extrato da amostra de sedimento (A) monodimensional; (B) amostra de sedimento fortificada com $500 \mu \mathrm{g} \mathrm{L} \mathrm{L}^{-1}$ de mistura dos padrões. 1) Triclorfom, 2) tricloroguaiacol (PI), 3) propanil, 4) fipronil, 5) propiconazol I e II, 6) trifloxistrobina, 7) permetrina cis e trans, 8) difenoconazol I e II e 9) azoxistrobina. As setas indicam os interferentes da matriz. que coeluem com os analitos na $1 D-G C$ 
Uma das técnicas mais empregadas para separação e análise quantitativa de agrotóxicos em sedimentos é a GC/MS no modo monitoramento de íon selecionado (SIM). Neste caso, mesmo no modo SIM, é possível que ocorram coeluições com interferentes que apresentam em sua estrutura os íons escolhidos para o monitoramento dos analitos. A separação em duas dimensões, proporcionada pela GCxGC, minimiza a possibilidade de coeluições. Em estudo realizado por Smalling e colaboradores ${ }^{37}$ para determinação de agrotóxicos em sedimento foram verificados os limites de detecção instrumentais para 85 agrotóxicos, por GC/ $\mu \mathrm{ECD}$ e GC/MS. Os resultados obtidos mostraram a grande sensibilidade da $\mathrm{GC} / \mu \mathrm{ECD}$, pois os limites de detecção foram mais baixos para essa técnica $\left(0,5\right.$ a $1 \mu \mathrm{g} \mathrm{kg}^{-1}$ para GC/ $\mu \mathrm{ECD}$ e 1 a $2 \mu \mathrm{g} \mathrm{kg}^{-1}$ para GC/MS).

A maior seletividade e capacidade de pico do sistema GCxGC proporcionaram a possibilidade de separação entre os analitos e os interferentes da matriz, fazendo com que seja possível o emprego da GCxGC com um detector seletivo, como o $\mu$ ECD para determinação desses compostos em sedimentos. Outra opção seria o uso de um detector universal, como o TOFMS, que permite deconvolução espectral nos casos de coeluição. Entretanto, o seu custo é ainda proibitivo para muitos laboratórios. Por outro lado, o custo de um GCxGC/ $\mu$ ECD se assemelha ao de um GC/MS. O $\mu$ ECD é um detector que apresenta alta sensibilidade e seletividade para a determinação de compostos halogenados. ${ }^{38}$ Essas importantes características de detecção quando aliadas ao potencial de separação da cromatografia gasosa bidimensional abrangente propiciam ótimos resultados de separação e detecção. Assim, os resultados obtidos até agora apontam para o desenvolvimento de um método analítico para determinação destes 8 agrotóxicos em sedimentos localizados em áreas próximas às lavouras de arroz.

\section{CONCLUSÕES}

A análise das amostras de sedimentos por GCxGC, devido à alta seletividade e capacidade de pico da técnica, possibilita a separação dos 8 agrotóxicos com menor probabilidade de interferência de constituintes da matriz. Através dos cromatogramas das amostras fortificadas com padrões dos agrotóxicos ficou evidente o maior potencial da técnica GCxGC (conjunto de colunas DB-5/DB-17ms) em relação à 1D-GC para a separação e quantificação dos compostos estudados. Em relação ao tempo de análise, as duas metodologias praticamente se equivalem, com 27,9 min para 1D-GC e 29,4 min para GCxGC.

Os resultados obtidos até agora apontam para a possibilidade de desenvolvimento de um método analítico para determinação destes 8 agrotóxicos, provenientes da orizicultura do Rio Grande do Sul, em sedimentos, através do emprego de $\mathrm{GCxGC} / \mu \mathrm{ECD}$, sem a necessidade de emprego de um detector de mais alto custo, como o TOFMS. Tal método poderá, futuramente, contribuir para o monitoramento de rotina da qualidade ambiental dos sedimentos localizados em sítios próximos à lavoura de arroz neste estado.

\section{AGRADECIMENTOS}

Ao Conselho Nacional de Desenvolvimento Científico e Tecnológico (CNPq), à Fundação Estadual de Proteção Ambiental (FEPAM) e à Coordenação de Aperfeiçoamento de Pessoal de Nível Superior (CAPES) pelo suporte financeiro a este projeto.

\section{REFERÊNCIAS}

1. IRGA; Lavoura Arrozeira 2008, 56, 1.

2. van der Hoff, R. G.; van Zoonen, P.; J. Chromatogr., A 1999, 843, 301.

3. Barceló, D.; Chromatographia 1988, 25, 928.
4. Hogendoorn, E.; van Zoonen, P.; J. Chromatogr., A 2000, 892, 435.

5. Blumberg, L. M.; David, F.; Klee, M. S.; Sandra, P.; J. Cromatogr., A 2008, 1188, 2 .

6. Phillips, J. B.; Beens, J.; J Chromatogr., A 1999, 856, 331.

7. Liu, Z. Y.; Phillips, J.; J. Chromatogr. Sci. 1991, 29, 227.

8. Adahchour, M.; Beens, J.; Vreuls, R. J. J.; Brinkman, U. A. Th.; Trends Anal. Chem. 2006, 25, 438.

9. Adahchour, M.; Beens, J.; Brinkman, U. A. Th.; J. Chromatogr., A 2008, 1186, 67.

10. von Mühlen, C.; Zini, C. A.; Caramão, E. B.; Marriott, P. J.; Quim. Nova 2006, 29, 765 .

11. von Mühlen, C.; Zini, C. A.; Caramão, E. B.; Marriott, P. J.; Quim. Nova 2007, 30, 682.

12. Pedroso, M. P.; Godoy, L. A. F.; Fidélis, C. H. V.; Ferreira, E. C.; Poppi, R. J.; Augusto, F.; Quim. Nova 2009, 32, 421.

13. Mondello, L.; Tranchida, P. Q.; Dugo, P.; Dugo, G.; Mass Spectrom. Rev. 2008, 27, 101.

14. Dallüge, J.; Beens, J.; Brinkman, U. A. Th.; J. Chromatogr., A 2003, $1000,69$.

15. Dallüge, J.; van Rijn, M.; Beens, J.; Vreuls, R. J. J.; Brinkman, U. A. Th.; J. Chromatogr., A 2002, 965, 207.

16. Banerjee, K.; Patil, S. H.; Dasgupta, S.; Oulkar, D. P.; Patil, S. B.; Savant, R.; Adsule, P. G.; J. Chromatogr., A 2008, 1190, 350.

17. Khummueng, W.; Trenerry, C.; Rose, G.; Marriott, P. J.; J. Chromatogr., A 2006, 1131, 203.

18. Bordajandi, L. R.; Ramos, L.; González, M. J.; J. Chromatogr., A 2006, $1125,220$.

19. Koritár, P.; Leonards, P. E. G.; de Boer, J.; Brinkiman, U. A. Th.; J. Chromatogr., A 2005, 1086, 29.

20. Panić, O.; Górecki, T.; Anal. Bioanal. Chem. 2006, 386, 1013.

21. http://www.irga.rs.gov.br/, acessada em Novembro 2008.

22. Grupo GERHI; Desenvolvimento de Ações para Implantação de Outorga na Bacia do Rio Santa Maria, 2006.

23. You, J.; Weston, D. P.; Lydy, M. J.; Arch. Environ. Contam. Toxicol. 2004, 47, 141

24. Cd room Agilent 6890N: Series Gas Chromatograph, 2001.

25. Godula, M.; Hajšlová, J.; Alterová, K.; J. High Resol. Chromatogr. 1999, 22,395 .

26. Grob, K.; Split and Splitless Injection for Quantitative Gas Chromatography, $4^{\text {th }}$ ed., Wiley: Weinheim, 2001.

27. Zhang, B.; Pan, X.; Venne, L.; Dunnum, S.; McMurry, S. T.; Cobb, G. P.; Anderson, T. A.; Talanta 2008, 75, 1055.

28. Jönsson, J. Â.; LCGC 2002, 20, 920.

29. Kristenson, E. M.; Korytár, P.; Danielsson, C.; Kallio, M.; Brandt, M.; Mäkelä, J.; Vreuls, R. J. J.; Blens, J.; Brinkman, U. A. Th.; J. Chromatogr., A 2003, 1019, 65.

30. Bordajandi, L. R.; Ramos, J. J.; Sanz, J.; González, M. J.; Ramos, L.; J. Chromatogr., A 2008, 1186, 312.

31. Hoh, E.; Mastovska, K.; Lehotay, S. J.; J. Chromatogr., A 2007, 1145, 210 .

32. Schure, M. R.; J. Microcol. Sep. 1997, 9, 169.

33. Peters, S.; Truyols, G. V.; Marriott, P. J.; Schoenmakers, P. J.; J. Chromatogr., A 2007, 1146, 232.

34. Adam, F.; Vendeuvre, C.; Bertoncini, F.; Thiébaut, D.; Espinat, D.; Hennion, M. C.; J. Chromatogr., A 2008, 1178, 171.

35. Adam, F.; Bertoncini, F.; Coupard, V.; Charon, N.; Thiébaut, D.; Espinat, D.; Hennion, M. C.; J. Chromatogr., A 2008, 1186, 236.

36. Pizzutti, I. R.; Vreuls, R. J. J.; Kok, A.; Roehrs, R.; Martel, S.; Friggi, C. A.; Zanella, R.; J. Chromatogr., A 2009, 1216, 3305.

37. Smalling, K. L.; Kuivila, K. M.; J. Chromatogr., A 2008, 1210, 8.

38. Koritár, P.; Haglund, P.; de Boer, J.; Brinkiman, U. A. Th.; Trends Anal. Chem. 2006, 25, 373. 